VIERTELJAHRSSCHRIFT FÜR

WISSENSCHAFTLICHE PÄDAGOGIK

FERDINAND SCHÖNINGH

94 (2018) 71-88
VIERTELIAHRSSCHRIFT FÜR WISSENSCHAFTLICHE PAOAGOGIK brill.com/vfp

\title{
Optimieren oder Bilden?
}

Medientheoretische Reflexionen

\author{
Volker Ladenthin
}

Rheinische-Friedrich-Wilhelms-Universität Bonn, Bonner Zentrum für

Lehrerbildung, Historische und Systematische Erziehungswissenschaft,

Poppelsdorfer Allee 15, D-53115 Bonn

v.ladenthin@uni-bonn.de

\begin{abstract}
Man, as a »deficiency creature« (Herder), relies on culture to become man. Media are objectifications of culture: But exactly how do they help and exactly what do they help with? To what extent do media enable or prevent man from becoming who he wants to be or wants to become? It asks to what extent an optimization or education of humans (1) by tool and media use, (2) by replacement of defective and inferior parts or (3) by fragmentation and training of sub-competencies succeed.
\end{abstract}

\section{Bildung und Identität}

»Das Moralische aber ist es, worauf, nach dem Zeugniß unsers innersten Bewußtseyns, Alles ankommt: und dieses liegt allein im Individuo, als die Richtung seines Willens. In Wahrheit hat nur der Lebenslauf jedes Einzelnen Einheit, Zusammenhang und wahre Bedeutsamkeit: er ist als eine Belehrung anzusehn, und der Sinn derselben ist ein moralischer.« (Schopenhauer 1991, S. 515) Lange vor der Diskussion um den schillernden Begriff der Identität setzt Arthur Schopenhauer auf den Begriff des >Lebenslaufs $<$, und diesen wiederum unterstellt er der »Einheit, (dem) Zusammenhang und (der) wahre(n) Bedeutsamkeit« (ebd.), insofern er Prozess der Belehrung ist, dessen Sinn freilich die Moralität ist.

Bildung bezöge sich in dieser Perspektive auf das zu lernende Verhältnis zur Welt, so wie sie sich historisch und gegenwärtig erkennen und bearbeiten lässt, auf den Umgang mit dem Mitmenschen und das Verhältnis, das ein Mensch zu sich selbst hat. So wird aus dem psychologischen oder soziologischen Begriff 
>Identität< ein pädagogisches Thema: Wie lernt ein Mensch, ein gültiges Verhältnis zu sich selbst aufzubauen? Wie lernt er, sich selbst gültig zu bestimmen? Wie lernt er Selbstbestimmung? Alles Handeln muss vom Einzelnen im Laufe seines Lebens verantwortet werden - nur von ihm: Das ist die geforderte Moralität. Die gelernte Beziehung zur Welt, zu den Mitmenschen und zu sich selbst ist Gegenstand von Erkenntnis und Aufgabe, sie muss sachlich richtig sein und sittlich verantwortlich. Es gibt also - aus pädagogischer Sicht - nur eine Identität für alle: Die richtige.

Aber das Verhältnis, das ein Mensch zu sich selbst hat oder bildet, unterliegt verschiedenen Bedingtheiten: Es unterliegt natürlichen Entwicklungen (Kindheit, Pubertät, Adoleszenz), gesellschaftlichen Vorstellungen, aber auch den sozialen und medialen Möglichkeiten. Im folgenden Text soll thematisiert werden, inwiefern Medien es ermöglichen oder verhindern, dass der Mensch zu dem werden kann, der er werden will oder soll. Dabei leiten die beiden Begriffe >optimieren< und >bilden $<$ den Gedankengang. ${ }^{1}$

\section{Mensch und Werkzeug}

Der Mensch ist ein Mängelwesen - diese vielzitierte Bemerkung des Soziologen Arnold Gehlen (1904-1976) hat eine lange Tradition (vgl. Gehlen 1974, S. 33f). Gehlen hatte seine Überlegungen im Anschluss an Ausführungen Johann Gottfried Herders (1744-1803) formuliert, der das Werkzeug (man kann auch sagen: die Kultur) als Kompensation biologischen Mangels verstand. Als Naturwesen wäre der Mensch nicht überlebensfähig - er ist durch und durch ein denkendes Wesen - aber den richtigen Gebrauch dieses Denkvermögens muss er, frei zu den Instinkten, lernen. Die Eigenheit des Menschen ist also seine Lern- und Vernunftfähigkeit, die sich von den Fähigkeitszuwächsen und dem Instinktverhalten der Tiere grundsätzlich unterscheidet. Im Hinblick auf die moderne Frage, ob der Mensch ein Wesen ist, das sich durch Maschinen ersetzen lässt, ist die Formulierung Herders überraschend, dass der Mensch die »feinste Mechanik und Meßkunst« seiner Sinne üben soll. Der Körper wird hier metaphorisch als Maschine verstanden. Aber auch Herder greift in seinen Überlegungen auf Vorbilder zurück, vermutlich auf jene Stelle, in der Platon (428-348) den Mythos von der Erschaffung des Menschen berichtet (vgl. Platon 1957, S. 62).

Der Mensch könne nichts so gut wie die anderen Lebewesen. Er bedürfe, damit es >ausgleichend< sei, eines besonderen Vermögens. Dieses Vermögen sei natur- (oder hier: gott-) gegeben, also geschichtslos - die Kultivierung des 
Vermögens aber erfolge in der Zeit. Die Kultivierung macht aus, was man Geschichte nennen kann: individuelle Lebensgeschichte oder die Geschichte der Menschheit. Der Mensch muss sich also kultivieren, um als Mensch überleben zu können. Mit Werkzeug, also mit Maschinen oder Medien, und mit Vernunft. Werkzeuge und Vernunft scheinen kosmologisch notwendig (>ausgleichend $<$ ) ohne Werkzeuge und Vernunft wäre der Mensch dem Tier unterlegen. Aber der Ausgleich ist zugleich gestört. Mit Werkzeug und Vernunft nämlich ist der Mensch dem Tier überlegen: »Eine Spinne verrichtet Operationen, die denen des Webers ähneln, und eine Biene beschämt durch den Bau ihrer Wachszellen manchen menschlichen Baumeister. Was aber von vornherein den schlechtesten Baumeister vor der besten Biene auszeichnet, ist, daß er die Zelle in seinem Kopf gebaut hat, bevor er sie in Wachs baut.« (Marx 1972, S. 193)

Der Mensch muss demnach seine Vernunft so einsetzen, dass er natürliche Mängel kulturell kompensieren kann - er muss also Werkzeuge erfinden. Mit dem Werkzeug optimiert er seine unzureichenden Organe und Naturanlagen: Freilich mit der Folge, dass er seine freie Natur nun diesem Willen wieder unterordnen muss und er paradoxerweise durch seine Freiheit erst recht unfrei wird. Die Dialektik der Aufklärung. Die Arbeit mit dem Werkzeug ist keineswegs mehr ein Spiel der eigenen Kräfte; dieses Spiel ist nur noch der Idee der Kunst vorbehalten, die aber dann doch nur noch im Schein Symbol der Freiheit wäre (vgl. Schiller 1981, S. 58). Im Arbeitsprozess jedoch, dessen Organisation aus dem freien Willen entstand, muss sich der Wille dem selbstgeschaffenen Prozess unterordnen.

Wie kann man sich nun diese in sich widersprüchliche Kultivierung vorstellen, in der zunehmende Nutzung von Freiheit zugleich die Zunahme von Abhängigkeit mit sich bringt? Gelingt eine Kultivierung, die diese Dialektik ernst- und daher aufnimmt, durch Optimierung des Menschen bei innovativem Werkzeuggebrauch? Durch den Ersatz von >defekten und minderwertigen Teilen $\prec$ ? Durch Fragmentierung?

\section{$3 \quad$ Gelingt eine Optimierung des Menschen durch verbesserten Werkzeuggebrauch?}

Gemeinhin versteht man ein Werkzeug als etwas, das der Mensch zu etwas benutzt. Es ist ihm dienlich. Er entscheidet, wie und wozu er es einsetzt: Einen Hammer zum Schlagen, eine Kneifzange zum Ziehen. Das Werkzeug ist vom Menschen so geformt, wie er es braucht. Dazu ist ein pädagogisches Programm abgeleitet worden. 


\subsection{Medienbildung}

Spricht man über die Wirkungen neuer Medien im pädagogischen Kontext, dann kommt recht schnell das Argument, dass es auf den richtigen Umgang ankomme: Die Menschen müssen lernen, mit den Medien richtig umzugehen.

» Zu guter Bildung im 21. Jahrhundert gehören IT-Kenntnisse und der souveräne Umgang mit Technik und Risiken (sic! ${ }^{2}$ V.L.) digitaler Kommunikation ebenso wie das Lernen mittels der vielen neuen Möglichkeiten digitaler Medien.<(Bundesministerin Johanna Wanka) [...] Deshalb ist es wichtig, frühzeitig den richtigen Umgang mit ihnen zu lernen, eine gesellschaftliche Auseinandersetzung darüber anzuregen und die Menschen beim Schritt in digitale Lernwelten zu begleiten. [...] Digitale Medien sind kein Selbstzweck. Damit die Bürgerinnen und Bürger digitale Medien verantwortungsbewusst nutzen können, bedarf es einer spezifischen >Digitalen Bildung«.«( (в МвF)

Das ist ein traditionelles, ein freundliches, liberales Modell - und wir alle haben ja auch gelernt, mit dem Fernsehen sozialverträglich (»leichter, besser und erfolgreicher«, ebd.) umzugehen. Aber es fragt sich, ob das Modell in seiner Liberalität und Zuversicht nicht zu kurz greift: Auch der intentionale Gebrauch eines Mediums zeitigt unbeabsichtigte Nebenfolgen.

Zwar formt der Mensch sein Werkzeug. Aber umgekehrt kann ein Werkzeug auch den Menschen formen: Man kann einen schweren Schmiedehammer nur benutzen, wenn man sich zuvor entsprechend Muskeln antrainiert hat. In diesem Fall formt das Werkzeug den Menschen, der es bedienen will. Die Freiheit der Erfindung unterwirft den Erfinder der Unfreiheit des Erfundenen. Zwar bedienen wir uns der Werkzeuge und beherrschen sie, machen sie uns untertan; gleichwohl formen uns die Werkzeuge, wenn wir sie bedienen - oder sogar, um sie bedienen zu können. Die Medien formen uns - unabhängig davon, wie wir mit ihnen umgehen wollen. Die benutzten Medien nötigen uns ein Verhalten $\mathrm{ab}$, das sogar oder womöglich in Widerspruch zu unseren Handlungsplänen stehen kann. Wir müssen mit den Medien handeln und sie schreiben uns vor, mit ihnen handeln zu müssen. Da sie zudem nur auf bestimmte Art zu bedienen sind, nötigen sie uns bestimmte Verhaltensweisen ab.

Das ist beileibe keine neue Erfahrung, auch wenn sie immer wieder vergessen wird, wenn es um die Durchsetzung neuer Medien geht. Schon Platon wies darauf hin, dass das Medium der Schrift die Menschen vom Erinnern und Memorieren entlastet; und Medien sind Werkzeuge.

Die Menschen machen Maschinen. Aber diese Maschinen machen dann aus uns Menschen etwas Neues, Anderes - und zwar etwas, was gar nicht mit der Aufgabe der Maschinen zusammenhängt. Die Folge eines Mediums kann etwas sein, was wir gar nicht wollten, als wir dieses Medium benutzten. 
Alle Medien zwingen uns zu Verhaltensweisen, über die wir willentlich gar nicht mehr vollständig entscheiden können. Dies geschieht immer dann, wenn die Medien bestimmte Eigenschaften von uns für ihren Gebrauch voraussetzen (also immer), und wenn sie sozial so dominant sind, dass wir ohne größere Nachteile ohne diese Medien nicht mehr auskämen.

Das liberale Modell, dass Menschen Werkzeuge für sich nutzen und daher gestalten, ist bestenfalls die halbe Wahrheit, vermutlich viel weniger - eben weil wir uns bestimmten Medien gar nicht mehr entziehen können. So betrachtet aber sind Medien nicht nur die Verlängerung menschlicher Organe, sondern sie formen auch den Menschen dahingehend, sich als Verlängerung der Medien zu verhalten und Teil der Maschine zu werden. Die Maschine trainiert den Menschen, sich ihren Bedürfnissen anzupassen - der Mensch wird zur Verlängerung der Maschinen. Maschinen stellen Medien dar, die nicht nur wir formen, sondern die auch uns formen. Ob wir geformt werden, entzieht sich unserem Willen, also gerade dem, was wir - nach Schopenhauer - ausbilden wollen. Wir sind die Objekte der Medien. Sie beherrschen uns. Sie formen uns. Welche Formkraft die Medien haben, kann man aus ihrer Bedienung ableiten, aus den Fähigkeiten die man anwenden muss, um sie zu bedienen. Im Fernsehen gelten das gezeigte Bild und das gesprochene Wort. Nichts ist von Dauer, sondern >Geschwätz von gestern<. Wer behauptet, man könne das Fernsehen doch ausschalten, ignoriert, dass er längst nicht mehr darüber entscheiden kann, ob und wann er ein Medium ignoriert. Das Ausschalten isoliert den Fernsehkonsumenten vom sozialen System. Sobald er am sozialen System teilhaben will (>Teilhabe < wird von PISA als grundlegendes Bildungsziel ausgegeben), sobald jemand Bürger unter Bürgern sein will, kann und darf er nicht abschalten, sondern muss dauernd online sein.

Mit rigiden Maßnahmen müssen Schulen im Alltag Gebrauch und Missbrauch des Handys unterbinden - weil sonst Schule ad absurdum geführt wird. Das ist längst kein Disziplinproblem, so wie man einst den Gebrauch des Taschenrechners in Mathematikarbeiten gestatten oder verbieten konnte. Wenn die digitale Armbanduhr zum Handy wird, wird jede disziplinarische Maßnahme gegen Handys wirkungslos.

Die Frage ist also nicht allein, $o b$ uns Medien formen. Diese Frage ist schlicht zu bejahen. Die Frage ist: Wie formen uns die Medien? Bleiben wir menschlich? Was ist menschlich? Was sind wir eigentlich - wie sind wir eigentlich - und wie weit kann man sich verformen, ohne die Identität des Menschen zu verlieren? Medien allein auf ihre Funktionalität hin zu bestimmen und in Bildungsprozesse einzufügen unterbietet das Wissen, das wir über Medien haben. Digitale Bildung »soll Menschen befähigen, sich als selbstbestimmte 
Persönlichkeiten in einer sich beständig verändernden Gesellschaft zurechtzufinden und verantwortungsvoll ihre eigenen Lebensentwürfe zu verfolgen.« (BмBF) Letzter Prüfstein ist nicht ihre Funktionalität; regulative Ideen sind der Begriff des Menschen und die Frage, was am Mediengebrauch >selbstbestimmt ist und was nicht, was >eigener Lebensentwurf ist und was mediengerechtes Verhalten.

\subsection{Die Dialektik der Medien}

Recht frühzeitig in der letzten Moderne hatte Jean Jacques Rousseau die epistemische Macht der alten und neuen Medien thematisiert. Er hat mit ihnen die der menschlichen Geschichte innewohnende eigene Dialektik der Aufklärung formuliert - indem er auf die ungeheure Wucht dieser medialen Entwicklung hingewiesen hat, die den Menschen zum Werkzeug macht: Das, was den Menschen ausmache, »entarte« unter seinen Händen. »Alles, was aus den Händen des Schöpfers kommt, ist gut; alles entartet unter den Händen des Menschen. (...) Er vermischt und verwirrt Klima, Elemente und Jahreszeiten. (...) Er erschüttert alles, entstellt alles - er liebt die Mißbildung, die Monstren.« (Rousseau 1963, S. 107f) Die Vernunft, ohne die der Mensch nicht Mensch sein kann, ist zugleich genau das, was den Menschen zerstört. Mary Shelley (1797-1851) hatte Rousseaus Diagnose vorerst zu Ende gedacht und sich vorgestellt, wie es wäre, wenn der Mensch nun zum neuen Prometheus (Platon) würde und einen Menschen nach dem derzeitigen Ideal erschaffe. Dr. Frankenstein wird zu dem Wissenschaftler, der diesen Versuch unternimmt. Im Vorwort zur dritten Auflage des Romans »Frankenstein or The Modern Prometheus« (vgl. Shelley 2013) schreibt Shelley:

»Ich sah das grässliche Trugbild eines Menschen ausgestreckt liegen, und dann, auf die Arbeit irgendeiner mächtigen Maschine hin, gab es plötzlich Lebenszeichen von sich und regte sich mit einer ungelenken, kaum lebensähnlichen Bewegung. Grauenvoll mußte es sein, denn höchst grauenvoll wäre die Folge jeder menschlichen Bemühung, das grandiose Werk des Weltenschöpfers nachäffen zu wollen. Das Ergebnis seiner Arbeit mußte den Künstler entsetzen; von Grauen gepackt würde er vor dem abscheulichen Werk seiner Hände flüchten.«

PRIESTER 2003, S. 107

Auch hier: Die Dialektik der Aufklärung im Mediengebrauch. Der neue Schöpfer, der neue Prometheus flüchtet vor seinem eigenen Werk. Zuvor hatte Rousseau dies als Wesen des Menschen beschrieben: »Nichts will er so, wie es die Natur gemacht hat, nicht einmal den Menschen. Er muß ihn dressieren wie 
ein Zirkuspferd. Er muß ihn seiner Methode anpassen und umbiegen wie einen Baum in seinem Garten.« (Rousseau 1963, S. 107f)

Aber es muss nicht so dramatisch sein. Am Beispiel von Gestik, Laut und Schriftzeichen zeigt Rousseau, wie die Menschen neue Medien erfinden und nutzen, weil sie ihnen einen Vorteil versprechen: So ermöglicht etwa die Schriftsprache gegenüber der Sprechsprache eine größere Genauigkeit und Reflexion. Aber wie jede Verbesserung im medialen Bereich ist auch diese, so Rousseau, immer mit einem Verlust erkauft. Es geht nicht um >Technik und Risiko<, sondern um Gewinn und Verlust. So ist die Schrift, die den Gedanken fixiert, ohne jene Emotionalität, die in der Stimme oft frei von Semantik aber mit Bedeutung transportiert wird. Ein Telefonat ist mehr und zugleich weniger als eine E-Mail. Keines der beiden Medien kann das andere vollständig ausgleichen. Zwar ersetzen sich die Medien wechselseitig in gewisser Hinsicht, aber mit einher gehen - ohne Zutun des Menschen - Verlust und Gewinn. Jede Übersetzung gewinnt etwas hinzu und lässt etwas zurück. Walter Benjamin wird von dieser gedanklichen Position seine Übersetzungstheorie begründen: Alles Sprechen sei Übersetzen - im rousseauschen Sinne: Jedes Sprechen gewinnt etwas hinzu und verliert etwas (Benjamin 2007b, S. 16f).

Medien sind daher keine Mittel, die man funktional und behutsam einsetzen und ersetzen kann. Es sind immer Übersetzungen. Der Computer im Unterricht dient nicht der Bildung, sondern er übersetzt eine alte Welt in eine neue Welt, die wir noch nicht genau kennen und die daher Bildung verlangt. Jedes Medium lässt nur eine begrenzte Wirklichkeit zu. Und jeder Gewinn an neuen Möglichkeiten ist immer, zugleich und unabänderlich mit einem Verlust vorhandener Möglichkeiten verbunden.

Das hat nun Folgen. Wenn wir Gesellschaften haben, in denen ein Medium vorherrschend ist, verengen sich die Zugänge auf Wirklichkeit und die Möglichkeiten, mit Wirklichkeit umzugehen. Neil Postman hat dies am Beispiel des Fernsehens aufgezeigt (vgl. Postman 2008): Im Fernsehen wird alles zur Unterhaltung. Wir gewinnen mit dem Fernsehen Aktualität und verlieren Bedeutsamkeit. Wir gewinnen optische Evidenz und verlieren gedankliche Begründung: Ein Bild vernichtet mehr als 1000 Worte. Fernsehen ist die Rückkehr zur oralen Kultur - mit all den Vorteilen und Begrenzungen, die die orale Kultur gegenüber der Schriftkultur hat (vgl. Ong 1987).

\section{$3 \cdot 3$}

\section{Medien und Methoden der Erkenntnis}

Dabei ist die erkenntnistheoretische Pointe, dass es überhaupt nur deshalb mehrere Medien und damit Deutungen geben kann, weil das zu Deutende als Wahrheit (in seinem Wahrsein) vorausgesetzt wird. Gerade die Vielheit der Sprachen verweist auf die Notwendigkeit, die eine Sprache zu denken. 
Wilhelm von Humboldt hatte geschrieben: »Da die Naturanlage zur Sprache eine allgemeine des Menschen ist und Alle den Schlüssel zum Verständniss aller Sprachen in sich tragen müssen, so folgt von selbst, dass die Form aller Sprachen sich im Wesentlichen gleich seyn und immer den allgemeinen Zweck erreichen muss." (Humboldt 1988, S. 651) Ohne diese vorausgesetzte eine Sprache, die festlegt, was überhaupt Sprache ist, könnte es gar keine Sprachen im Plural geben. »Das Wahrsein«, notiert Walter Benjamin, »(das als solches natürlich unerkennbar ist) hängt mit der Unendlichen Aufgabe zusammen. Es ist aber nach dem Medium zu fragen, in welchem Wahrsein und Wahrheit im Zustande der Ungeschiedenheit sind. Welches ist dieses neutrale Medium?« (Benjamin 2007a, S. 70) Es kann ja keine historische Sprache sein, sondern es muss jene sein, die historisch (linguistisch) nachweisbare Sprachen möglich macht.

Ironischerweise bestätigen gerade die Konstruktivisten (allerdings indirekt) diese vorauszusetzende Einheit des Wahrseins, wenn sie von der Konstruiertheit aller menschlichen Auffassungen sprechen; denn diese Aussage selbst soll ja eben nicht wieder nur Konstrukt sein und durch ein anderes Konstrukt in Frage gestellt werden. Da ist man sehr empfindlich. Bei der Methode gibt es schon richtig und falsch: Dogmatismus lehnt der Konstruktivismus grundsätzlich ab. Er möchte das >Echte<.

Man kann diese systemimmanent ausweglosen Aporien des Konstruktivismus auch am Sprachgebrauch ablesen. In einem Neujahrsinterview sagte Bernhard Pörksen: »Der Konstruktivismus war als Medizin gegen den Dogmatismus gedacht, nicht als Modephilosophie für akademische Sektierer.« (Pörksen 2017) Es gibt also eine Intention, die nicht verhandelbar ist? Eine Absicht, die man völlig anerkennen muss, weil man sie sonst missversteht? »Als neue Heilslehre steht er traurig da. [...] Wir müssen echte Skepsis unterscheiden von Pseudo-Skepsis. « (ebd.) Wie begründet man das Kriterium der Unterscheidung, wenn der Rezipient die Unterscheidung nicht anerkennt? Ist das nicht auch Interpretation? »Zu echter (sic! V.L.) Skepsis gehört Demut, ein ernstes Hinterfragen der eigenen Position. Die Pseudo-Skepsis ist blind für die eigenen Konstruktionen. Sie stellt nur die anderen in Frage. Diese dafür umso mehr.« (ebd.) Es fragt sich nun, welcher Erkenntnisart das >Hinterfragen < angehört, das die »eigene Position « (ebd.) dann doch nicht als letzte eigene Position ausmachen kann, sondern es wie eine fremde Position betrachtet? Aus welcher Perspektive? Mithin müsste es eine eigene Position und dann eine noch eigenere Position geben, eine, die die eigene Position hinterfragt. Mit welcher Methode fragt die noch eigenere Position? Auch mit dem Konstruktivismus? Dann wäre sie aber auch wieder zu hinterfragen usw. Das eine des nunmehr 
geteilten Selbst könnte dem anderen des geteilten Selbst (das Ego dem Alter Ego) nicht trauen und das gemeinsame Ich der beiden Selbste müsste sich in sich selbst anzweifeln - bis in die Endlosigkeit stets unbegründeter Sätze.

Aber es gebe intern in uns ein echtes, eigentliches Wahrheitskriterium jedenfalls nach Pörksen. Auf die Frage des Redakteurs, welcher von zwei völlig unterschiedlichen Weltbeschreibungen wir denn folgen sollen, antwortet er: »Wir müssen nicht immer konsequent einer von beiden sein. Manchmal mag die liebevolle, >blinde Zuwendung die angemessene sein. Manchmal die pragmatische Ehrlichkeit. Wir müssen wählen.« (ebd.) Wer ist wir? Wie bestimmen wir das, was >wir <ist? Wonach müssen wir wählen? Wenn wir wählen können, muss es ein Kriterium geben, an dem wir unsere Wahl ausrichten? Was ist das Kriterium der richtigen Wahl? Das fragt (sich) auch der Redakteur: »(sz:) Was heißt das konkret?(Pörksen:) Erstens muss man die andere Wirklichkeit zu verstehen versuchen. Zweitens gilt es sich fragen: Kann ich die Motive dahinter nachvollziehen, also Verständnis zeigen? Und der dritte Schritt ist: Bin ich einverstanden? Also mag ich die Welt, die so entsteht? Oder lehne ich sie ab? « (ebd.) Woher aber weiß ich, ob und wann ich nachvollziehen kann? Zu Recht nachvollziehen kann? Was ist >Verständnis<? Und woher weiß ich, ob ich einverstanden bin? Was heißt >mögen<? Ist die Welt von dem abhängig, was ich mag, und was ich nicht mag? (Angenommen, jemand mag keine Konstruktivisten - darf er sie dann mit Erlaubnis der Konstruktivisten ignorieren? Also gibt es sie nicht? Ist, was der Fall ist, die Welt ein Geschmacksurteil zwischen dem, was ich mag und was ich nicht mag?) Wieso kann ich mir meiner selbst sicher sein, wenn ich nach dem entscheide, was ich mag? Weil diese Fragen so neu nicht sind, bietet auch Pörksen am Ende eine Empfehlung an, die ebenfalls so ganz neu nicht ist (aber vielleicht doch aus gerade diesem Grunde richtig?): ((sz:)Was also dagegen tun?(Pörksen:) Es braucht Aufklärung jenseits von Belehrung. Und dazu gehört, dass man selbst sagt und möglichst transparent begründet, wie man zu seiner Wahrheit und Wirklichkeitsauffassung gelangt ist.« (ebd.) Man mag vielleicht fragen: Was sonst?

Bildung als Identität des Menschen kann nun weder einfach und epistemologisch naiv als >Teilhabe beschrieben werden (weil sich das Ganze, an dem ein Mensch >Teil< haben soll, gar nicht formulieren lässt) noch als Opposition (weil jede Opposition immer schon an dem teilnimmt, wogegen sie opponiert: Die Kritik der Erkenntnis ist notwendigerweise eine Erkenntnis - die Verweigerung des Mediums ist immer medial artikuliert und daher vermittelt). Sie ist nicht >Ansichtssache< oder Konstruktion, zugleich aber auch nicht zu Ende oder endgültig. Bildung ist eine Aufgabe. Sie ist die Idee, sich ins Verhältnis zu 
Teilhabe und Opposition zu setzen, mithin Lernen als unendliches Übersetzen zu verstehen.

Folgende Fragen ergeben sich, um herauszufinden, welche Bedeutung ein Medium für den Identitäts- und Bildungsprozess hat: Zuerst wären die neuen Medien nicht als (funktionale) Werkzeuge, sondern als (ambivalente) Medien zu verstehen. Dann ist zu fragen: Was sind die spezifischen Fähigkeiten, die man braucht, um das Medium zu bedienen; welche Fähigkeiten verkümmern? Die Differenz und Identitätserfahrung: Was ist epistemische Eigenart (Gewinn und Verlust) eines Mediums? In welchen gesellschaftlichen Verknüpfungen (Voraussetzung, Folgen und Konstitution) realisiert sich ein Medium? In welcher Beziehung steht ein Medium zu dem stets vorausgesetzten, aber nie formulierbaren Ganzen des Wahrseins (Benjamin) und des Menschseins (Humboldt)?

4 Gelingt eine Optimierung des Menschen durch Fragmentierung?

In der Schule wird z. B. die Feinmotorik so optimiert, dass man lernt zu schreiben. Das lückenhafte Wissen wird ergänzt, vervollständigt. Das Denken wird optimiert, damit man regelhaft denken kann. Hier realisiert sich das alte Wissen, »daß an einer Stelle ausschließlich erzeugt werden muß, was in reichem Maße entstehen soll« (Comenius 196o, Kap. 8). Im Begriff der Kompetenz taucht dieser Gedanke der Optimierung durch Fragmentierung wieder auf und zwar in besonderer Form, die überraschen mag. Denn Kompetenzen werden stets im Plural abgehandelt. Welche Konzepte man auch wählt, nie geht es um die Kompetenz, sondern um die Kompetenzen oder es werden Komposita genannt, denen der Oberbegriff fehlt. Kompetenzen sind wie Werkzeuge, wie ein spezielles Organ im Gehirn, das optimiert werden soll. In der Regel werden drei Kompetenzen genannt: Sachkompetenzen, Sozialkompetenzen und Ichkompetenzen.

Was aber bildungstheoretisch interessiert: Was steht da in der Mitte? Was passiert, wenn sich die Ellipsen überschneiden? Wer sorgt für die Überschneidung und wer regelt sie? Wie genau ergibt sich aus sozialen, methodischen und fachlichen Kompetenzen »Handlungskompetenz«? Durch Mischung? Paritätisch? Durch den Goldenen Mittelweg? Paart sich hier Exzellenz-Psychologie mit Spruchweisheiten?

Wer handelt da eigentlich? Ergibt sich Handlungskompetenz aus der Summe der Einzelkompetenzen? Ist das ein Automatismus? Wo bleibt aber da unser Wille, der nach Schopenhauer doch das einheitsstiftende Moment ist? 
Was ist >Problemlösekompetenz ? Es fragt sich doch: Wer löst welche Probleme? Wer bestimmt mit welcher Kompetenz die zu lösenden Probleme? Wer macht sie sich warum zu eigen? Wessen Probleme sind es, die wer lösen soll? Und wozu lösen wir die Probleme? Damit sie gelöst sind? Soll man alle Probleme lösen, die sich stellen - und wenn nein: Wer bestimmt die Reihenfolge und die Auswahl der zur Lösung anstehenden Probleme? Und schließlich und grundsätzlich: Wie heißt und wo lernt man die Kompetenz, die zu lösenden Probleme zu bestimmen? Die Kompetenztheorie erweist sich als biometrische Antwort auf Frankensteins Traum: Verbesserung des Menschen durch Optimierung von Teilfähigkeiten: »Die Zerlegung des Menschen in seine Fähigkeiten ist eine Projektion der Arbeitsteilung auf deren vorgebliche Subjekte, untrennbar vom Interesse, sie mit höherem Nutzen einsetzen, überhaupt manipulieren zu können.« (Adorno 1951, S. 76)

Der Befund ist, dass Kompetenztheorien zwar einzelne Fähigkeiten des Menschen optimieren wollen, jedoch keine Überlegungen zum Zusammenhang dieser Teilkompetenzen angestellt werden. Es ist daher eine Fähigkeit notwendig, die die optimierten Teilleistungen zu einem sinnvollen Ganzen zusammenzuführen vermag: »Es liegen viele Keime in der Menschheit, und nun ist es unsere Sache, die Naturanlagen proportionierlich zu entwickeln (...) und zu machen, daß der Mensch seine Bestimmung erreiche. (Kant 1983, S. 701) Was aber diese >Bestimmung « ist, das klärt keine der gängigen Kompetenztheorien. Sie bleiben defizitär. D.h. sie greifen in den »Lebenslauf« (Schopenhauer) ein, ohne ihren Eingriff anders als dadurch zu begründen, dass man in einen Lebenslauf eingreifen und Teilleistungen optimieren kann. Sie stören einen gelebten Zusammenhang, ohne einen neuen zu bieten, anzubieten oder wenigstens anzuregen. Kompetenzen zu schulen ist, als würde bei einem Kind der Schnuller durch eine Schere ersetzt werden, um anschließend zu beobachten, was passiert.

So gehört es sicherlich zur Sozialkompetenz, sich jener Kleidung und Körpergestaltung zu bedienen, die allgemein anerkannt und sozial belohnt wird wozu zum Beispiel ein gebräunter Körper gehört (vgl. Paris 1985). Gleichzeitig erwirbt man aber im Biologieunterricht die Sachkompetenz, zu wissen, dass einem mit UV-Strahlen belasteten Körper ein erhöhtes Krebsrisiko innewohnt (vgl. DIE WELT 2016). Nach welcher Regel soll man nun zwischen der Sozialkompetenz (>gebräunter Körper signalisiert Gesundheit und Gepflegtheit, Erfolg und soziale Integration<) und Sachkompetenz (>Sonnenstrahlen erhöhen das Hautkrebsrisiko<) vermitteln?

Wem dieses Beispiel zu lebensweltlich ist und zu wenig auf die heutige Schule bezogen, dem wäre die Frage zu stellen, wie jemand zu entscheiden 
hätte, der zwischen Durchsetzungsvermögen als Selbstkompetenz und Teamgeist als Sozialkompetenz zu entscheiden hätte? Wann gilt was? Oder der in der Schule erstaunt feststellt, dass die soeben erworbene Sozialkompetenz >Teamgeist< bei der Klausur unerwünscht ist? Nur - warum ist das so? Die Regel, nach der so entschieden wird, die lernt er nach der Kompetenztheorie nicht. Diese Kompetenz ist nicht vorgesehen. Sie steht nicht auf dem Lehrplan.

Kompetenztheorien gehen davon aus, dass sich die aufgeführten Kompetenzen in ihren Segmenten schon sirgendwie< sinnvoll ergänzen und sich zu einem >sinnvollen< Ganzen fügen. Woher rührt dieser Optimismus in einer Epoche, die von Beginn an durch Streit (Kant), Widersprüche (Marx), Antagonismen (Adorno) oder Widerstreit (Lyotard) beschrieben wurde (vgl. Colpe 1999, S. 21-27)? In der Kompetenztheorie wird all dies zu lösbaren >Problemen< harmonisiert, deren Lösung immer schon als gut angesehen wird. Jede Lösung scheint besser als keine.

Es stellen sich die Fragen: Wie wird jene Fähigkeit benannt, die die einzelnen Kompetenzen zusammenführt und reguliert? Wie ist das Zusammenspiel der Teilkompetenzen zu denken? Wer entscheidet darüber, wann welche Kompetenzen anzuwenden sind? Welche Konsequenzen für das Ich hat die Herausbildung von Einzelkompetenzen? Wie kann diese Auswirkung kontrolliert reflektiert werden?

\section{Gelingt eine Optimierung des Menschen durch Ersatz von} >defekten und minderwertigen Teilen $<$ ?

Stiftungen, die sich in die Bildungspolitik einmischen wollen, sprechen gerne davon, dass sie etwas >gestalten ${ }^{3}$ wollen<; also etwas in eine Gestalt bringen, mithin formen oder gar bilden wollen. Die Metaphorik hat eine aufschlussreiche Tradition. In Thomas Manns Erzählung »Das Gesetz« (Mann 1960; Mann 2003) berichtet der Erzähler über Moses, dass er die Lust zur »Gestaltung « des Volkes habe »wie der Steinmetz Lust hat zu dem ungestalten Block, woraus er feine und hohe Gestalt, seiner Hände Werk, zu metzen gedenkt« (Mann 196o, S. 810). In Anklang an Langbehns Rembrandtbuch ${ }^{4}$ nimmt Thomas Mann die Vorstellung vom Erzieher als Künstler auf und legt Volksbildung als künstlerisches Wirken, als Formung und eben Gestaltung einer >Rohmasseく aus. »Ich [...] komme nun zum Eigentlichen, der Gesetzgebung, die ich als eine Art michelangeleskem Skulpturwerk an einem Rohmaterial von Volkskörper behandle.« (Mann 1979, S. 298) 
Moses sei von »Bildnerlust « (Mann 1960, S. 819) bestimmt - ein Wort, das die metaphorische Wurzel von Bildung und Bildnis aufnimmt. Er sei in »all sein[em] Denken und Trachten darauf beschränkt gewesen, seines Vaters Geblüt in der Absonderung für sich allein zu haben, um es zu bilden und ungestört aus der heillosen Masse, die er liebte, eine heilige Gottesgestalt zu metzen « (ebd., S. 835).

Wie Biomasse gerät diesem Volkserzieher das Volk zum Objekt des »Bohrens, Wegsprengens und Formens« (ebd., S. 840), »damit er vor allem einmal das Gehudel zu Gott bilde und etwas Heilig-Anständiges, ein reines Werk, dem Unsichtbaren geweiht, daraus haue (ebd., S. 836). Bildung sei die »Lust« am Volke »zu [...] seiner Gestaltung« (ebd., S. 824), »Bildnerlust« (ebd., S. 819) in dem Sinne, dass der »ungestalte Volksleib « (ebd., S. 846), ein »bloßer Rohstoff [...] aus Fleisch und Blut« (ebd., S. 847), ein »Klotz« (ebd., S. 847) in jene »Formung « (ebd., S. 847) gebracht werde, die dem »Bildner des Volks« (ebd., S. 850) vorschwebt: »Wie es aussah in dem Gehudel, und wie sehr es ein bloßer Rohstoff war aus Fleisch und Blut, dem die Grundbegriffe der Reinheit und Heiligkeit abgingen; wie sehr Mose von vorn anfangen und ihnen das Früheste beibringen mußte, das merkt man den notdürftigen Vorschriften an, mit denen er daran herumzuwerken, zu meißeln und zu sprengen begann - nicht zu ihrem Behagen; der Klotz ist nicht auf des Meisters Seite, sondern gegen ihn, und gleich das Früheste, was zu seiner Formung geschieht, kommt ihm am allerunnatürlichsten vor.« (ebd., S. 847)

Bildung als Wegsprengen und Ersatz minderwertiger Teile? Ist das nicht die moderne Idee der Optimierung des Körpers?

Seit je kann die Medizin Teile des Menschen amputieren oder ersetzen. Unzweifelhaft helfen Operation und Prothesen dem Menschen, ein würdiges Leben zu führen: Ohne eine neue Linse könnte ich diesen Text nicht schreiben. Leitidee ist der funktionstüchtige, letztendlich der gesunde Mensch. Er ist die Leitidee des hippokratischen Eides: Alles zu tun, was der Gesundheit dient. Aber Optimierung meint heute: Das Gesunde zu verbessern. Das betrifft z. B. Schönheitsoperationen. Aber selbst hier mögen medizinische Gründe oder psychische Erkrankungen wie mangelndes Selbstbewusstsein die Ursache sein: Die entwicklungsbedingte Unfähigkeit, sich selbst zu akzeptieren. Sich zu akzeptieren, wie man ist. Die Phase der Selbstfindung ist noch nicht abschlossen. Noch bestimmt ein Fremdbild den Wert des Selbstbildes. Aber geht es nicht auch bei medizinisch verordneten Schönheitsoperationen um mehr? Geht es nicht darum, herauszufinden, wer man reigentlich $<$ ist? Mit den gesteigerten Operationsmöglichkeiten stellen sich ein philosophisches und ein pädagogisches Problem: Machen wir uns oder sind wir? Müssen wir uns 
verstehen oder entwerfen? Müssen wir uns formen, gestalten oder bilden? Aber wer sind wir selbst, die wir lernen, uns zu verändern, anzupassen oder treu zu bleiben? Und wie viel können wir von uns selbst ersetzen, ohne nicht mehr wir selbst zu sein? Bleiben wir dieselben, wenn wir uns selbst ersetzten? Kommen wir zu uns selbst, oder verlieren wir uns? Wie bestimmen wir uns indem wir uns nur bestätigen? Oder indem wir uns auslegen, oder neu schaffen? Einen arithmetischen Mittelweg gibt es nicht. Vielleicht aber eine sinnvolle Konzeption. Eine Konzeption des sinnvollen Entwurfs der eigenen Identität. Die Leitidee ist nicht die Optimierung, sondern die Angemessenheit. Das Leben soll nicht optimiert werden, sondern gelingen. Wann aber gelingt das Leben? Wenn es sinnvoll ist? Was aber ist sinnvoll? Dass wir uns so nehmen, wie wir sind, ist zuerst unausweichlich: Wir können die Zeit nicht bestimmen, in die hinein wir geboren wurden. Wir konnten unsere Eltern nicht bestimmen, unser Geschlecht, unseren Körper, unsere Nationalität, unsere Religion. Die schlichte und blinde Faktizität unserer körperlichen Verfassung ist ein Teil unseres Lebenssinns. In diesem Sinne sind wir, was wir sind. Andererseits ist unser Leben nicht vorherbestimmt. Wir können uns zu unseren Eltern verhalten. Wir können unser Vater-Land verlassen, zur Mutter-Sprache eine Sprache lernen, die uns bisher fremd und unbekannt war. Wir können uns zu unserem Geschlecht kulturell verhalten. Wir können unsere Herkunft bedenken - und obwohl wir nie ganz frei sind von Sinnvorgaben und Sinnfestlegungen durch unsere Lebensgeschichte, haben wir doch die innere Freiheit, uns zu ihr ins Verhältnis setzen zu können. In dieser Hinsicht erschaffen wir uns durch das, was wir tun und geben, unserem Leben einen Sinn. Welchen Sinn aber? Woher wissen wir, was sinnvoll ist?

Lebenssinn ergibt sich nicht aus Einzelzwecken; vielmehr provozieren Einzelzwecke die Frage danach, ob sie denn sinnvoll sind. Man kann allerdings nicht danach fragen, ob es sinnvoll ist, nach Sinn zu fragen: Denn dieses Fragen setzt die Sinnhaftigkeit des Fragens schon voraus. Sinn mag daher zwar selbsttätig erkannt werden, aber kann er auch selbstermächtigend bestimmt und zuvor gelernt werden? »Während (nun) Techné lehrbar und erlernbar ist [...], gilt für das Wissen und die Vernunft, die die praktische Lebenssituation des Menschen erhellen und leiten, das genaue Gegenteil.« (Gadamer 1967b, S. 167f) Sinn wäre demnach nicht lehrbar, weil er schon immer da ist, wenn wir uns entschließen, Lernen als sinnvoll zu erachten. Wir leben also, wenn wir begründet handeln, immer schon im Horizont von Sinnunterstellungen (vgl. Marquard 1986). Diese Sinnunterstellungen wären allen Zwecksetzungen immer schon vorausgesetzt, auch dann, wenn wir nichts von ihnen wissen und kein Wort für sie haben (vgl. Gadamer 1967b). Wir müssten nicht in allem nach 
unserer Bestimmung suchen, weil wir immer schon zu etwas bestimmt wären. Wir lebten immer schon in einem Sinnhorizont, wenn wir anfangen, nach ihm zu suchen.

Allerdings gilt auch das Axiom moderner Anthropologie, dass der Mensch frei ist, dass die einzige Bestimmung des Menschen die sei, sich selbst zu bestimmen. Damit kann ihm kein Lebenssinn verliehen werden, sondern er muss ihn selbst stiften (vgl. Sartre 1968). Der Mensch sei >Werk seiner selbst $<$ (Pestalozzi), weil er andernfalls keine Verantwortung für sein Handeln übernehmen könnte. Der Mensch macht die Geschichte - das ist die Idee der Aufklärung seit der Antike. Die Natur hat keine Geschichte, sie hat nur Entwicklung. Alles Denken lohnt also nur, wenn wir auch gemäß dem eigenen Denken handeln können.

Wie immer man die Konstitution von Sinn auch diskutieren mag - als Hermeneutik des Seins (vgl. Gadamer 1967a) oder als spontanen Akt einer voraussetzungslosen Existenz (vgl. Sartre 1964): Immer verweist die Struktur des vernünftigen Handelns auf die Notwendigkeit einer gültigen Bestimmung von Lebenssinn als »Aufgabe « (Benjamin). Man muss Sinn bestimmen, wenn man verantwortungsvoll und begründet handeln will - also im Hinblick auf Bildung: »So wie sich nämlich mit der zunehmenden Bestimmtheit alles Ungebildete dem Gebildeten nähert; so nähert sich auch, mit der zunehmenden Zufälligkeit, das Gebildete immer mehr dem Ungebildeten. Denn der Begriff des Unorganisierten ist mit dem Begriff des Zufälligen unzertrennlich verknüpft.« (Moritz 1962, S. 97)

So lassen sich Bildungsprozesse vielleicht durch folgende Frage erschießen: Gibt es Unabänderliches, dessen Sinngehalt auszulegen ist? Welche Handlungsoptionen und Folgen ergeben sich aus dem, was wir wissen? Welche dieser Handlungsoptionen dienen einem universalen guten Leben? Welche dieser Handlungsoptionen lassen sich sittlich rechtfertigen?

Es ist das, was Humboldt als Grundfrage bezeichnet hatte, und Schopenhauers Maxime personal auslegt: »Die letzte Aufgabe unsres Daseyns: dem Begriff der Menschheit in unsrer Person, sowohl während der Zeit unsres Lebens, als auch noch über dasselbe hinaus, durch die Spuren des lebendigen Wirkens, die wir zurücklassen, einen so großen Inhalt, als möglich zu verschaffen, diese Aufgabe löst sich allein durch die Verknüpfung unsres Ichs mit der Welt zu der allgemeinsten, regesten und freiesten Wechselwirkung. Diess allein ist nun auch der eigentliche Massstab zur Beurtheilung der Bearbeitung jedes Zweiges menschlicher Erkenntniss. « (Humboldt 1903, S. 282ff) Das ist mehr und anders als systemimmanente Optimierung von Teilleistungen, wie sie derzeit als Ideal für die Schule ausgegeben wird. 


\section{Literatur}

Adorno, Theodor W. (1951): Ich ist Es. In: Minima Moralia. Reflexionen aus dem beschädigten Leben. Hrsg. von Theodor W. Adorno. Frankfurt/Main: Suhrkamp, S. 75-77.

Benjamin, Walter (2007): Fragmente zur Sprachphilosophie und Erkenntniskritik (1918-1921). In: Kairos. Schriften zur Philosophie. Ausg. und mit einem Nachw. vers. von Ralf Konersmann. Frankfurt/Main: Suhrkamp, S. 68-78. [= Benjamin 2007a]

Benjamin, Walter (2007): Über Sprache überhaupt und über die Sprache des Menschen. (1916). In: Kairos. Schriften zur Philosophie. Ausg. und mit einem Nachw. vers. von Ralf Konersmann. Frankfurt/Main: Suhrkamp, S. 7-22. [= Benjamin 2007b]

Colpe, Carsten (1999): Weltdeutungen im Widerstreit. Berlin, New York: De Gruyter.

Comenius, Johann A. (1960): Grosse Didaktik [1657]. Hrsg. und übers. von Andreas Flitner. Düsseldorf, München: Küpper.

Gadamer, Hans-Georg (1967): Die Universalität des hermeneutischen Problems. In: Kleine Schriften. I. Philosophie. Hermeneutik. Hrsg. von Hans-Georg Gadamer. Tübingen: Mohr Siebeck, S. 101-112. [= Gadamer 1967a]

Gadamer, Hans-Georg (1967): Über die Planung der Zukunft [1965]. In: Kleine Schriften. I. Philosophie. Hermeneutik. Hrsg. von Hans-Georg Gadamer. Tübingen: Mohr Siebeck, S. 161-178. [= Gadamer 1967b]

Gehlen, Arnold (1974): Der Mensch. Seine Natur und seine Stellung in der Welt (10. Aufl.). Frankfurt/Main: Athenaion.

Herder, Johann Gottfried (1961): Ideen zur Philosophie der Geschichte der Menschheit [1784-1791]. In: Menze, Clemens (Hrsg.): Johann Gottfried Herder - Humanität und Erziehung. Paderborn: Schöningh, S. 95-135.

Humboldt, Wilhelm von (1903): Theorie der Bildung des Menschen. Bruchstück [1793]. In: Gesammelte Schriften (Bd. I). Hrsg. von Albert Leitzmann. Berlin: B. Behr's.

Humboldt, Wilhelm von (1988): Werke in fünf Bänden (Bd. III: Schriften zur Sprachphilosophie.). Hrsg. von Andreas Flitner/Klaus Giel. Darmstadt: Klett-Cotta.

Kant, Immanuel (1983): Über Pädagogik [1803]. In: Immanuel Kant:Werke in zehn Bänden (Bd. 10). Hrsg. von Wilhelm Weischedel. Darmstadt: Wiss. Buchgesellschaft, S. $697-712$.

Mann, Thomas (1960): Das Gesetz [1944]. In: Thomas Mann: Gesammelte Werke in zwölf Bänden (Bd. VIII). Frankfurt/Main: S. Fischer.

Mann, Thomas (2003): Das Gesetz. Novelle [1944]. Mit Kommentaren von Volker Ladenthin/Thomas Vormbaum. Berlin, Boston: De Gruyter.

Mann, Thomas (1979): Brief an Agnes E. Meyer vom 17. November 1943. In: Thomas Mann. Briefe 1937-1947. Hrsg. von Erika Mann. Frankfurt/Main: S. Fischer.

Marquard, Odo (1986): Zur Diätetik der Sinnerwartung [1983]. In: Odo Marquard (Hrsg.): Apologie des Zufälligen. Philosophische Studien. Stuttgart: Philipp Reclam Junior, S. 33-53. 
Marx, Karl (1972): Das Kapital. Kritik der politischen Ökonomie (Bd. I). Berlin: Dietz Verlag.

Moritz, Karl Philipp (1962): Die Signatur des Schönen. Inwiefern Kunstwerke beschrieben werden können? In: Schriften zur Ästhetik und Poetik. Kritische Ausgabe. Hrsg. von Hans Joachim Schrimpf. Tübingen: Niemeyer, S. 93-103.

Ong, Walter J. (1987): Oralität und Literalität. Die Technologisierung des Wortes. Opladen: Westdt. Verlag.

Paris, R. (1985): »Schön«. Warum wir in der Sonne braten. Oder: Die soziale Bedeutung der Sonnenbräune. In: Frankfurter Rundschau vom 18. Juli 1985.

Platon (1957): Protagoras [o.J.]. In: Sämtliche Werke. (Bd. I). Hrsg. von Ernesto Grassi unter Mitarbeit von Walter Hess; in der Übers. von Friedrich Schleiermacher. Hamburg: Rowohlt, S. 49-96.

Pörksen, Bernhard (2017): Die Wahrheit ist umkämpfter denn je. Medienwissenschaftler Pörksen über 2017, das Jahr der Fake News (Interview von Friedemann Karig). In: Süddeutsche Zeitung vom 29. Dezember 2017.

Postman, Neil (2008): Wir amüsieren uns zu Tode. Urteilsbildung im Zeitalter der Unterhaltungsindustrie [1985]. Frankfurt/Main: Fischer.

Priester, Karin (2003): Mary Shelley. Die Frau, die Frankenstein erfand. München: Blanvalet.

Rousseau, Jean-Jacques (1963): Émile oder über die Erziehung [1762]. Hrsg. von Martin Rang. Stuttgart: Reclam.

Sartre, Jean-Paul (1964): Die Transzendenz des Ego. Drei Essays. Hamburg: Rowohlt.

Sartre, Jean-Paul (1968): Drei Essays. Mit einem Nachwort von Walter Schmiele. Frankfurt/Main: Ullstein.

Schiller, Friedrich (1981): Über die ästhetische Erziehung des Menschen. Text, Materialien, Kommentar. Hrsg. von Wolfgang Düsing. München, Wien: Hanser Verlag.

Schopenhauer, Arthur (1991): Die Welt als Wille und Vorstellung [1819]. In: Arthur Schopenhauers Werke in fünf Bänden (Bd. II). Hrsg. von Ludger Lütkehaus. Zürich: Haffmans.

Shelley, Mary Wollstonecraft (2013): Frankenstein oder Der moderne Prometheus/ Frankenstein Or The Modern Prometheus [1818]. Hrsg. und übers. von Alexander Pechmann. München: dtv Verlagsagesellschaft.

\section{Internetquellen}

Bundesministerium für Bildung und Forschung (вмвғ): Bildung digital. URL: https://www.bmbf.de/de/bildung-digital-3406.html (Datum des letzten Abrufs: 03. Januar 2018). 
DIE WELT: Eine gesunde Sonnenbräune gibt es nicht. Veröffentlicht am 29.06.2016. URL: https://www.welt.de/gesundheit/article156665014/Eine-gesunde-Sonnenbrae une-gibt-es-nicht.html (Datum des letzten Abrufs: 29. Dezember 2017).

\section{Endnoten}

1 Der Text geht zurück auf einen frei gehaltenen Vortrag auf einer Tagung des »bibor« (Bonner evangelisches Institut für berufsorientierte Religionspädagogik) am 26.06.2014 zum Thema »Der optimierte Mensch« an der Universität Bonn und auf eine erste Schriftfassung unter dem Titel: Identität. Der optimierte Mensch - oder der gebildete Mensch? In: PädagogikUNTERRICHT. Die Fachzeitschrift für die pädagogische Fächergruppe 35, 1/2015, S. 24-32, wurde aber für diese Veröffentlichung gekürzt, erweitert und überarbeitet. ...müsste es nicht »Technik und Umgang« oder »Chancen und Risiken« heißen?

3 Bei der Bertelsmann-Stiftung z. B. gibt es gleich eine ganze Reihe von Broschüren mit immer der gleichen Metaphorik: »Projekt Vielfalt leben - Gesellschaft gestalten«, »Projekt In Vielfalt leben - Zusammenhalt gestalten «, »Projekt Migration fair gestalten «, »Stadt mit Vielfalt für alle gestalten «, »Unsere Ganztagsschulen in Deutschland fair gestalten «, »Digitalisierung ist unaufhaltsam. Wir müssen sie jetzt gestalten!«, »Zukunft gestalten bedeutet heute in Bildung investieren«, »Unsere Organisation: Handeln und gestalten « usw. (über 800 Nachweise), URL: https://www .bertelsmann-stiftung.de/de/system (Datum des letzten Abrufs: 05 . Januar 2018).

4 o.A. (1890): Rembrandt als Erzieher. Von einem Deutschen. Vgl. die kritische Interpretation von Mann, Klaus: Ein wirklicher Vorläufer. In: Pariser Tageblatt vom 9. Februar 1936, zit. nach: Mann, Klaus (1993): Zahnärzte und Künstler. Aufsätze, Reden, Kritiken 1933-1936. Hrsg. von Uwe Naumann und Michael Töteberg. Reinbek bei Hamburg: Rowohlt, S. $384 f f$. 